\title{
The role of selection in driving landscape genomic structure of the waterflea Daphnia magna
}

\author{
LUISA ORSINI, ${ }^{*}$ JOACHIM MERGEAY, ${ }^{*} \dagger$ JOOST VANOVERBEKE* and LUC DE MEESTER* \\ *Laboratory of Aquatic Ecology, Evolution and Conservation, University of Leuven, Ch. Deberiotstraat 32, 3000 Leuven, \\ Belgium, $†$ Research Institute for Nature and Forest, Gaverstraat 4, B-9500 Geraardsbergen, Belgium
}

\begin{abstract}
The combined analysis of neutral and adaptive genetic variation is crucial to reconstruct the processes driving population genetic structure in the wild. However, such combined analysis is challenging because of the complex interaction among neutral and selective processes in the landscape. Overcoming this level of complexity requires an unbiased search for the evidence of selection in the genomes of populations sampled from their natural habitats and the identification of demographic processes that lead to present-day populations' genetic structure. Ecological model species with a suite of genomic tools and well-understood ecologies are best suited to resolve this complexity and elucidate the role of selective and demographic processes in the landscape genomic structure of natural populations. Here we investigate the water flea Daphnia magna, an emerging model system in genomics and a renowned ecological model system. We infer past and recent demographic processes by contrasting patterns of local and regional neutral genetic diversity at markers with different mutation rates. We assess the role of the environment in driving genetic variation in our study system by identifying correlates between biotic and abiotic variables naturally occurring in the landscape and patterns of neutral and adaptive genetic variation. Our results indicate that selection plays a major role in determining the population genomic structure of D. magna. First, environmental selection directly impacts genetic variation at loci hitchhiking with genes under selection. Second, priority effects enhanced by local genetic adaptation (cf. monopolization) affect neutral genetic variation by reducing gene flow among populations and genetic diversity within populations.
\end{abstract}

Keywords: ecological genomics, environmental association analysis, environmental genomics, founder effects, genetic drift, monopolization hypothesis, redundancy analysis

Received 7 March 2012; revision received 8 October 2012; accepted 11 October 2012

\section{Introduction}

The integration of ecology and genomics allows us to gain insights into the processes by which populations respond and adapt to their environment. Such an integration is accomplished in two ways: through ecological and evolutionary studies on traditional genetic model species that have well-developed genomic tools (Drosophila melanogaster, Caenorhabditis elegans and Mus musculus) and through the application of molecular genetics and genomics to organisms with well-understood

Correspondence: Luisa Orsini, Fax: +32 016320771;

E-mail: luisa.orsini@bio.kuleuven.be ecologies [e.g. Gasterosteus aculeatus (Jones et al. 2012); Arabidopsis lyrata (Turner et al. 2010); Daphnia pulex (Colbourne et al. 2011)]. Traditional genetic model species offer a staggering amount of molecular biological knowledge, but their ecologies are often poorly understood. Although this is changing (e.g. Balanya et al. 2006), it remains that these species have not been selected for their ecological role and therefore are not ideal to answer ecological questions. For a number of species that play a central role in natural ecosystems (e.g. keystone species), we have a good understanding of their ecologies and associated environmental data, but, so far, limited access to genetic and genomics tools. Although this situation is rapidly changing owing to the 
higher accessibility of next-generation sequencing technologies (Wang et al. 2009; Metzker 2010; Davey et al. 2011), in recent years nonmodel taxa have been mostly investigated in 'forward genetics' genome scan studies (Nosil et al. 2009). Genome scan studies identify genome regions under selection, either directly or more often through linkage, through the screening of several loci (in the order of hundreds, Storz 2005). The obvious advantage of a forward genetics approach is the analysis of populations in their natural habitats. The disadvantage for the majority of nonmodel species is the lack of genomic tools that would allow functional genomics follow-up studies (Luikart et al. 2003). Linking outlier loci with candidate genes is confined to species with known genomes or transcriptomes, and therefore, most genome scans studies remain exploratory (but see Bonin et al. 2006; Namroud et al. 2008; Orsini et al. 2012). While ecological model species will lag behind genetic model species for functional genomics studies for the years to come, they are best suited for establishing a link between genomic and environmental variation (Eckert et al. 2010). Establishing this link may be one of the more promising ways to understand processes and mechanisms driving present-day population genomic structure in the wild.

Here we investigate the waterflea Daphnia magna Straus, an emerging genomic model system and a renowned ecological model system, to identify adaptive and neutral processes that lead to present-day landscape genomic structure. Daphnia magna is a keystone species in lakes and ponds (Lampert 2011). It has been extensively used as a model in evolutionary biology (Hairston et al. 1999; Cousyn et al. 2001; Ebert 2005; Decaestecker et al. 2007; Van Doorsaler et al. 2009) and to study adaptive response of ecological traits to the environment (reviewed in Miner et al. 2012). A draft annotated genome is now available for D. magna and genomic tools are increasingly becoming available (Routtu et al. 2010; Jansen et al. 2011; Orsini et al. 2011). Yet, we still face the challenge of linking genome architecture with ecological important traits.

Differential heterogeneous selection at the genome level has been shown in natural populations of $D$. magna in response to three strong ecological gradients (Orsini et al. 2012) known to exert strong selection pressure and to induce microevolutionary response in this species [predation (Cousyn et al. 2001), parasitism by the endoparasite Pasteuria ramosa (Decaestecker et al. 2007) and land-use intensity (Michels 2008; Coors et al. 2009)]. Here we build on these results and study how the complex mosaic of biotic and abiotic variables naturally occurring in the landscape, in addition to the already studied gradients, contributes to driving neutral and adaptive genomic variation. For this analysis we used the same genotypes analysed in Orsini et al. (2012) for microsatellite and Single Nucleotide Polymorphism (SNP) markers. In addition, we measured genetic variation at the mitochondrial gene COI and collected data for twenty biotic and abiotic variables naturally occurring in the landscape. Using three types of neutral markers (mtDNA, microsatellites and SNPs), we study past and current demographic patterns to infer demographic processes that determine present-day population genetic structure in the wild. We identify the likely processes, demographic and selective, responsible for driving landscape genomic structure in D. magna. As compared to the genome scan study of Orsini et al. (2012) which focused on environmental gradients exerting strong selection pressure (fish predation, land-use intensity and parasite infection), here, we try to understand not only how these specific selection pressures drive population genomic structure as compared to neutral processes, but also how a wide array of biotic and abiotic variables contribute to such structure and interact with space, in an attempt to interpret the complex processes occurring in natural landscapes.

\section{Materials and methods}

\section{Population sampling}

We analysed populations of D. magna (Table S1, Supporting information) from nineteen shallow ponds in Belgium (Fig. 1B). Individuals from each population were collected from the surface layer of the dormant egg bank. Waterfleas are cyclical parthenogens that alternate parthenogenetic or clonal reproduction with sexual reproduction, commonly occurring at the end of the growing season. The latter produces dormant eggs that accumulate in the sediment forming egg banks. We analyse animals hatched from the resting egg bank, sampling the genetic diversity after sexual reproduction, minimizing the confounding effects determined by fluctuations in the frequency of clones (genotypes) in the active population during the parthenogenetic phase. The pool of clones that contribute to the next generation best represents changes in genetic composition across generations (years) (Vanoverbeke \& De Meester 2010). By sampling only the superficial layer of the egg bank we avoid confounding factors potentially determined by the overlap of multiple sexual generations. The populations studied in the present study were used in a genome scan analysis that identified candidate genome regions linked with three environmental stressors known to exert strong selection pressure (Orsini et al. 2012) [predation (Cousyn et al. 2001), parasitism of the endoparasite Pasteuria ramosa (Decaestecker et al. 2007) and land-use intensity (Michels 2008; Coors et al. 2009)]. Of the 19 ponds, nine were sampled from the coastal 
(A)

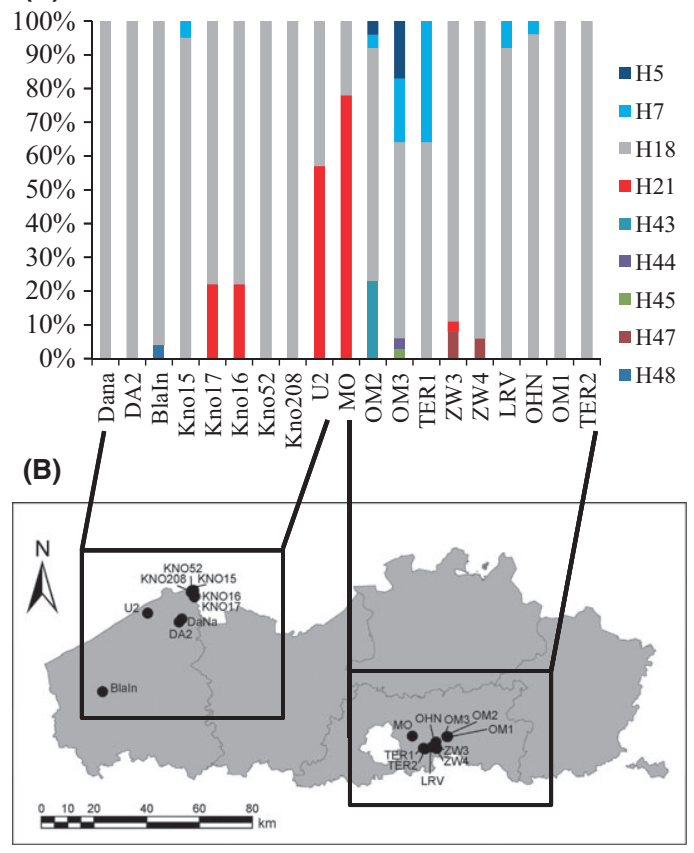

(C)

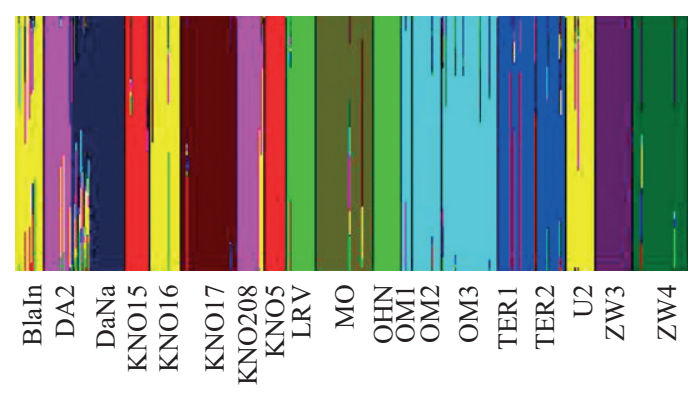

(D)

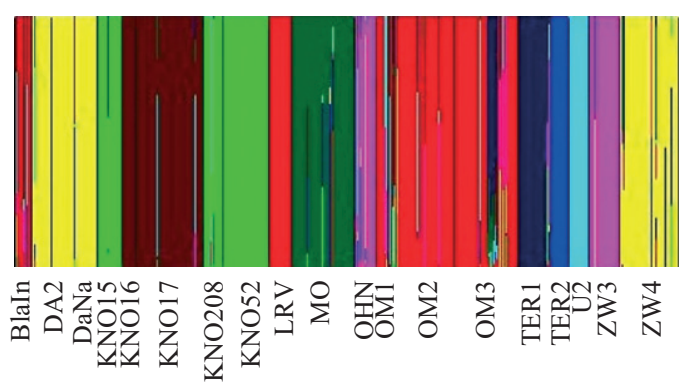

Fig. 1 Population genetic structure analysis. (A) COI mtDNA haplotype frequency and distribution at regional scale in Belgium in the set of 19 populations analysed. The correspondence between the geographic location of the populations and the haplotype frequency is shown (B). Population genetic structure of the 19 populations for microsatellites (C) and SNPs (D) inferred with the software BAPS is also shown.

region and 10 from the central region of Belgium (inland, region of Leuven, Table S1, Supporting information). The sediment was filtered and ephippia (dormant eggs) hatched according to standard protocols (Onbe 1978; Marcus 1990). Clonal lineages were established from successful hatchlings, kept in climate rooms $\left(20{ }^{\circ} \mathrm{C}\right.$ and $16: 8 \mathrm{~h}$ photoperiod) and fed unicellular algae Scenedesmus obliquus. Twenty environmental variables were scored at the time of sampling (Table S1, Supporting information). For further details on the sampling design and handling of clonal lineages see Orsini et al. (2012).

\section{Genetic markers}

In a previous genome scan analysis, 84 microsatellites $(N=576)$ and 82 SNPs $(N=578)$ were genotyped (Orsini et al. 2012). Microsatellite loci are evenly distributed across the chromosomal map of D. magna (Routtu et al. 2010), providing an unbiased representation of the genome. The SNPs developed from EST libraries (Orsini et al. 2011) are also randomly distributed in the genome. The genome scan analysis (Orsini et al. 2012) identified 27 loci (17 SNP and 10 microsatellites) linked with general stress response, 13 loci uniquely linked each with one of the three stressors (2 SNP and 2 microsatellite loci were associated with fish predation; three SNPs and one microsatellite were associated with land-use intensity, and five SNPs were associated with parasite infection), and 126 (71 microsatellites and 53 SNPs) neutral loci. The background ecological knowledge of the natural system where the populations occur allowed identifying populations along orthogonal gradients of selection for the three stressors. In the genome scan, all combinations of the three factors (presence/absence of fish; high / low prevalence of the parasite Pasteuria; highlow land-use intensity) were analysed using at least two replicates for each contrast. Genetic variation linked with both specific (either predation, parasitism or land use) and general stress responses (across the three stressors) was identified. The patterns identified in the spatial survey were validated in time, using sediment cores with known paelolimnological history for fish predation (Cousyn et al. 2001), land-use intensity (Coors et al. 2008; Michels 2008) and parasite infection (Decaestecker et al. 2007). For fish predation, patterns identified in the spatial survey were also validated in experimental evolution trials. Building on the genome scan analysis, here, we study the patterns of genetic variation at both neutral markers and markers hitchhiking with genes under selection to analyse the impact of neutral (demographic) and selective processes on natural 
populations of D. magna. In addition, we sequenced the partial sequence of the mtDNA COI gene from 479 individuals of the 19 study populations (accession numbers to GenBank: JQ279821-JQ280299). For each population we sequenced 20-36 individuals, with the exception of three populations for which 6,12 and 17 individuals were sequenced, respectively (Table S1, Supporting information). The partial sequence of the mtDNA COI gene was obtained using different combinations of four amplification and sequencing primers (two universal primers and two specific primers designed for this study, Table S2, Supporting information), as sequence variation in the primer sites sometimes hampered the amplification and/or sequencing of the target gene. For the PCR amplification of the partial COI, 20-30 ng of genomic DNA was amplified in a touch down (TD) PCR consisting of $1 \mu \mathrm{M}$ of each primer, $2.5 \mu \mathrm{m}$ of each of the dNTPs, $1 \mu \mathrm{M}$ of $\mathrm{MgCl}_{2}$, and $0.2 \mathrm{U}$ of Silverstar Taq Polymerase (Eurogentec, Belgium). All amplifications were performed in $20-\mu \mathrm{L}$ final volumes using a T1 PCR machine (Biometra, Germany). TD PCR cycling conditions were as follows: five cycles consisting of denaturation at $94{ }^{\circ} \mathrm{C}$ for $1 \mathrm{~min}$, annealing at $45^{\circ} \mathrm{C}$ for $1.5 \mathrm{~min}$ and extension at $72{ }^{\circ} \mathrm{C}$ for $1.5 \mathrm{~min}$ were followed by 35 cycles of $94{ }^{\circ} \mathrm{C}$ for $1 \mathrm{~min}, 50^{\circ} \mathrm{C}$ for $1.5 \mathrm{~min}$ and $72{ }^{\circ} \mathrm{C}$ for $1.5 \mathrm{~min}$. PCR products were purified using nucleofast 96 PCR purification kit (Mackerey-Nagel, Germany) and doublestrand sequenced using Big Dye terminator chemistry on an ABI PRISM 3031 (Applied Biosystems), following the manufacturer instructions. Sequence assembly and editing were done using SeqScape v2.5 (Applied Biosystems). Prior to the analysis, sequences were aligned with Clustal W (Thompson et al. 1994) in Bioedit 7.0.1 (Hall 1999). In Table 1, we summarize the total set of analyses performed and described in detail in the following.

\section{Genetic analysis: assessing past and current demographic history}

Historical events such as range expansion, bottlenecks and founder events can complicate the interpretation of the effects of contemporary natural selection across heterogeneous landscapes and obscure current patterns of gene flow and drift (e.g. Hutchinson \& Templeton 1999; Duvernell et al. 2008). We studied patterns of neutral genetic variation using markers with different mutation rates to infer the demographic processes that may have influenced the genetic structure of the study populations. We also studied patterns of variation at loci hitchhiking with genes under selection (in the following, we refer to these loci as describing adaptive variation). The detailed analysis of neutral and adaptive variation com- bined with the past genome scan analysis (Orsini et al. 2012) provides an integrated picture of the neutral and selective processes driving population genomic structure in our study system.

$m t D N A$. Phylogenetic analysis of the COI mtDNA haplotypes was performed to assess to what extent the ancient (e.g. last ice age) demographic history of D. magna at European scale may have influenced the recent demographic history at the regional scale (Belgium). We reconstructed the phylogeny of Daphnia species, including our D. magna sequences and COI sequences of $D$. magna available in NCBI at the time of the analysis (Table S3, Supporting information; mostly De Gelas \& De Meester 2005). We used as outgroups both closely related species $(D$. similis Claus and cryptic sibling species) as well as distantly related species (D. pulex Leydig and cryptic sibling species) to D. magna, following the most recent phylogeny of freshwater zooplankton (Adamowicz et al. 2009). The phylogenetic inference was conducted using Bayesian statistics and maximum likelihood approaches (see Supplementary methods). The ID assigned to the COI mtDNA haplotypes followed the scheme already adopted by De Gelas \& De Meester (2005). Identical haplotypes to the ones already published were given the same haplotype code for consistency (Table S3, Supporting information).

To study the effect of regional founder events during the postglacial colonization history of D. magna in Europe, we estimated changes in effective population size $\left(N_{\mathrm{e}}\right)$ and studied the distribution of COI at the European scale (mismatch distribution). To document changes in the regional effective breeding population size $\left(N_{\mathrm{e}}\right)$ through time, we used the Bayesian skyline plot drawn in Beast (Drummond \& Rambaut 20022006) including all COI mtDNA haplotypes from Europe (mostly De Gelas \& De Meester 2005). The theoretical expectation is that $N_{\mathrm{e}}$ changes over time in the presence of population expansion following for instance a founder or a bottleneck event (Drummond et al. 2005). To study postglacial population expansion at the European scale, a mismatch distribution was calculated for the European haplotypes treated as a single population using Arlequin v. 3.1 (Excoffier et al. 2005). The mismatch distribution analysis describes the distribution of pairwise nucleotide differences among haplotypes based on a model of sudden population expansion (Schneider \& Excoffier 1999). The distribution is usually multimodal in samples drawn from populations at equilibrium, as it reflects the highly stochastic shape of gene trees, but it is usually unimodal in systems not in equilibrium, where populations have passed through a recent bottleneck (Slatkin \& Hudson 1991; Rogers \& 


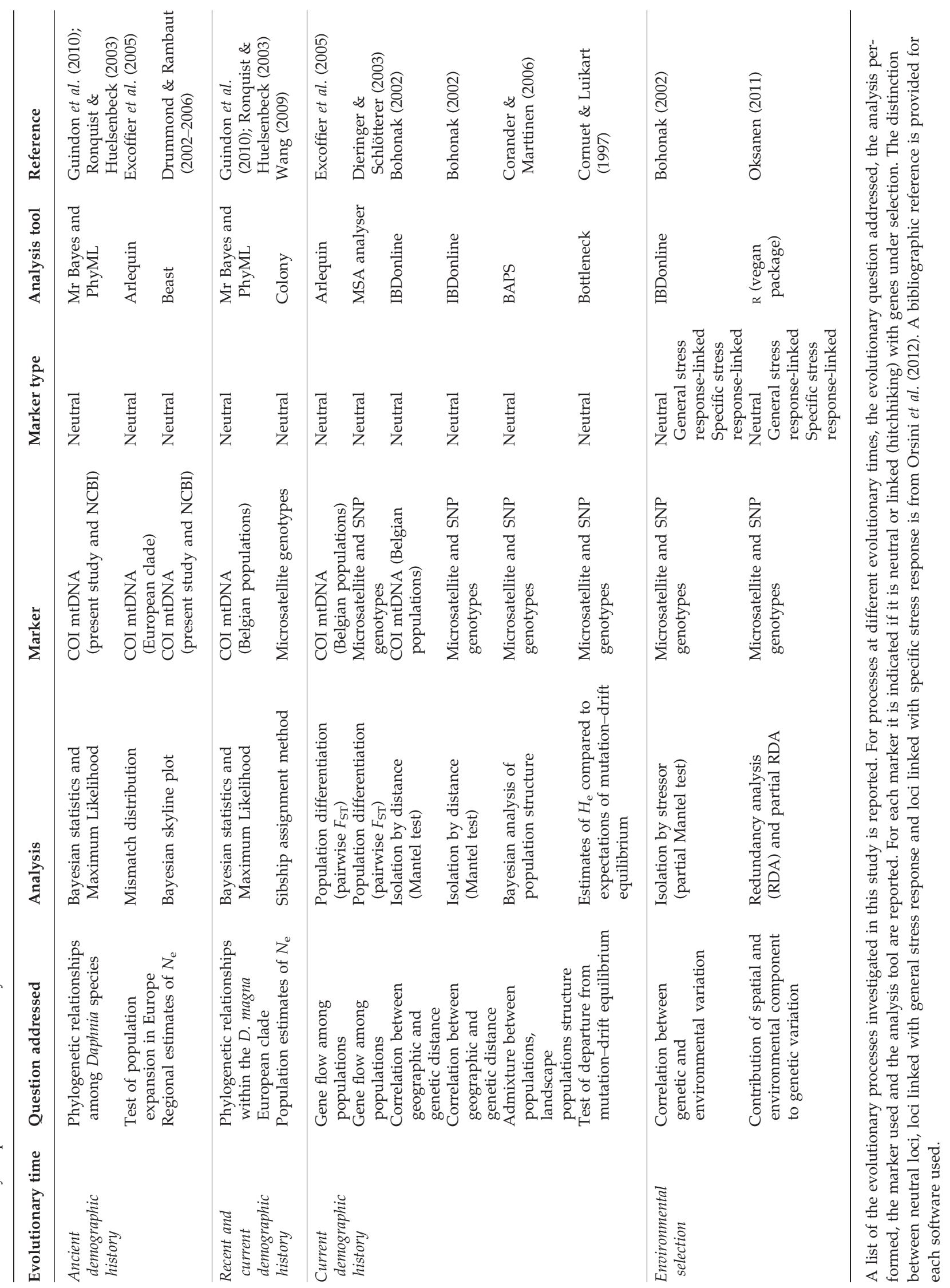


Harpending 1992) or through a range expansion with high levels of migration between neighbouring demes (Ray et al. 2003). The statistical significance of the estimated mismatch parameters was tested using a sum of square deviations (SSD) test of goodness of fit, comparing observed and expected mismatch distributions. We calculated 95\% confidence intervals with 1000 replicates using the Arlequin's parametric bootstrap approach.

After the phylogenetic position of the Belgian haplotypes was established within the phylogeny of daphnids and signals of postglacial colonization of Europe analysed, we focused on a detailed analysis of the Belgian populations to infer recent demographic history. In these analyses, we used only haplotype identity for mtDNA, as mutations at mtDNA are negligible for the study of current demography. Haplotype statistics were inferred with DNAsp (Librado \& Rozas 2009). We calculated genetic differentiation (pairwise $F_{\mathrm{ST}}$ ) using Arlequin v. 3.1 (Excoffier et al. 2005) and inferred pattern of isolation by distance using the software IBD online (Bohonak 2002).

Neutral microsatellites and SNPs. For the analysis of microsatellite and SNP markers we capitalized on the previous genome scan analysis in which a distinction was made among loci under selection for one of the focal environmental gradients (parasites, fish or land use), loci underlying general stress response (loci linked with more than one strong environmental gradient analysed) and loci that behaved neutrally (Orsini et al. 2012). We used the genotype data for the markers that behaved neutrally to infer current demographic processes driving population genetic structure in D. magna. For each population 16-52 individuals were genotyped, with the exception of three populations for which only 10, 12 and 13 individuals were genotyped for SNP loci (Table S1, Supporting information). To infer population structure and admixture, we used the Bayesian analysis implemented in BAPS (Corander et al. 2004, 2008) separately for neutral microsatellite and SNP loci and performed a nonspatial genetic mixture analysis using individuals and populations as basic units to be clustered. Different starting values of $K(2-25)$ were used to verify the robustness of the results. Pairwise $F_{\mathrm{ST}}$ was calculated with MSA analyser (Dieringer \& Schlötterer 2003). We tested for $\mathrm{H}-\mathrm{W}$ equilibrium per population over all neutral loci and gametic linkage disequilibrium between each pair of neutral loci using Genepop online (Rousset 2008) with 10000 and 1000 permutations, respectively. Patterns of IBD were previously inferred for these markers (Orsini et al. 2012).

For each population we estimated current effective population size using the software Colony (Wang 2009). Colony estimates $N_{\mathrm{e}}$ using the sibship assignment method described in Wang (2009). In brief, estimations of $N_{\mathrm{e}}$ are derived in terms of the frequencies of a pair of offspring taken at random from the population being sibs sharing the same one or two parents. SNPs are generally less useful than microsatellites in detecting effective population size, because the systematic removal of alleles determined by reduction in effective population size leads to monomorphism in SNPs more often than in microsatellites (Morin et al. 2004). Therefore, we here only estimate values of $N_{\mathrm{e}}$ obtained from microsatellites. In addition to the estimates of current $N_{\mathrm{e}}$, we used the software Bottleneck version 1.2.02 (Cornuet \& Luikart 1997; Luikart \& Cornuet 1998) to test for departure from mutation-drift equilibrium in our study populations. 'Bottleneck' compares the observed and the expected levels of heterozygosity with respect to expectations for a population at mutation-drift equilibrium (number of heterozygotes with Hardy-Weinberg equilibrium expectation, Cornuet \& Luikart 1997). A systematic excess of expected heterozygosity across loci is interpreted as a signature of reduction in $N_{\mathrm{e}}$. This is generally interpreted as signature of a recent bottleneck or founder event (Luikart \& Cornuet 1998). However, here we use this analysis, in parallel with the mismatch distribution analysis, as a mean to test for departure of our populations from a mutation-drift equilibrium. For this analysis both microsatellite and SNP multilocus genotypes were analysed using an infinite allele model (IAM). The IAM provides a more liberal test for heterozygosity excess, as it does not take into consideration the effect of size homoplasy among microsatellite alleles. Significance of deviations from mutation-drift equilibrium was assessed with a two-tailed Wilcoxon test.

\section{Environmental association analysis}

We performed an environmental association analysis testing for significant correlations between 20 abiotic and biotic environmental variables as measured in the habitats (Table S1, Supporting information) and neutral and adaptive genetic variation. With adaptive genetic variation we refer to genetic variation at loci linked with specific or general stress response in the previous genome scan analysis (Orsini et al. 2012). The environmental association analysis was performed using classic population genetics methods (c.f. partial Mantel test) as well as canonical redundancy analysis (RDA). The latter is commonly used to estimate the relative contribution of spatial and environmental variation structuring communities (e.g. Declerck et al. 2011; De Bie et al. 2012) but it has been recently applied in a population genetic context (Legendre \& Fortin 2010; Vangestel et al. 2012). 
Partial mantel tests. As exploratory tool, a partial Mantel test was performed between all pairs of environmental variables to identify covariation in the landscape, correcting for geographic distance. In addition, a modified isolation by distance analysis (Bohonak 2002) was employed to measure the degree of correlation between genetic divergence among populations and divergence in different environmental variables to which these populations are exposed in the natural landscape, correcting for geographic structure (partial Mantel test, 'Isolation by Stressor', see Orsini et al. 2012). This analysis was conducted for neutral marker, loci linked with general stress response and loci linked with specific stress response (either fish predation, land-use intensity or parasite infection). This analysis is directly comparable to the analysis conducted in Orsini et al. (2012) for microsatellite and SNP loci in relation to the three strong environmental gradients (fish predation, land use and parasites).

$R D A$. As performing multiple pairwise tests between genetic and environmental variables may inflate type I error, we also performed the environmental association analysis using RDA. RDA allows to disentangle the relative contribution of environmental and spatial components in driving landscape genomic structure.

Redundancy analysis is a multiple linear regression method between a first matrix of dependent and a second matrix of independent (explanatory) variables (Legendre \& Legendre 2012). The dependent matrix in our analysis was represented by genotypic data (or a derived set of principal coordinate variables of pairwise genetic distances, PCoA), whereas the explanatory variables were expressed either as a spatial matrix $S$ ( $X$ and $Y$ coordinates or derived distance-based Moran's eigenvector maps, dbMEM), an environmental matrix $E$ (or derived sample loadings of principal component analyses, PCA) or both.

The dependent variables were sample loadings of PCoA using Nei's genetic distances (Nei 1972) calculated with Genetix 4.05 (Belkhir et al. 2004). For microsatellite and SNP loci, the following categories of markers were used: (i) neutral loci, (ii) loci linked with general stress response, (iii) loci linked with fish predation pressure, (iv) loci linked with landuse intensity, and (v) loci linked with parasite infection (only for SNPs, as the previous genome scan analysis did not identify microsatellite outlier loci linked with parasite infection, Orsini et al. 2012). In addition, an RDA was performed using as dependent variable COI mtDNA-haplotype composition per pond. These data were Hellinger transformed to make them suitable for RDA analysis (Legendre \& Gallagher 2001).
The explanatory variables of the RDA were the environmental variables measured in the habitat. To reduce the number of explanatory environmental variables, prior to the RDA, we performed a PCA of the environmental variables expressed in multiple classes to identify the major axes of variation. This was done because the adjusted coefficient of multiple regression declines as more variables are included in the global model (Borcard et al. 2011). Since we have 19 ponds, divided over two geographic clusters, we need to constrain the number of explanatory variables a priori. The environmental variables that were PCA-reduced are the parasite and the land-use variables. Land use was previously expressed in three distinct categories: arable land, pastures and mixed semi-natural cover (Orsini et al. 2012). When these categories were plotted using a PCA, the first land-use axis was represented by variation in pastures, whereas the second axis was represented by variation in arable land (positive PCA loadings) and mixed semi-natural cover (negative PCA loadings). Similarly, four classes of parasites were previously measured in the ponds analysed: Amoebidium parasiticum, Binucleata daphniae, Pasteuria ramosa and Vorticella sp. When the abundance of the four parasites was plotted using a PCA, the first axis was represented by $A$. parasiticum, whereas the second was a combination of the other three parasites (all positive PCA loadings). To assess the role of spatial variation and unmeasured spatially structured environmental variation, we constructed distance-based Moran's eigenvector maps (MEM) and included them as explanatory variables in the environmental association analysis. Because our populations are clustered in two main groups and this may result in an underestimation of diversity within the clusters (Declerck et al. 2011), we constructed a staggered matrix with separate MEM variables for the coastal (MEM1) and the inland data set (MEM2-4), as described in Declerck et al. (2011). Next to these MEM variables, a dummy variable (coast versus inland) was used. In addition to the global analysis that involved all the populations in the study system $(N=19)$, we also performed RDA separately for the two regional groups of inland and coastal populations (Table S1, Supporting information). This was done to account for different environmental characteristics and selection pressures exerted by the environmental gradients in these two population clusters.

Depending on the hypothesis tested, different sets of E (environment) and S (space) were used. When specific hypotheses were tested regarding the effect of a priori selected environmental variables, we performed RDA according to the double stop criterion of Blanchet (Blanchet et al. 2008), which consist of performing forward selection only when the global model (containing all 
variables) is significant. This procedure avoids inflation of the overall type 1 error and the inclusion of too many variables in the model (for details see Borcard et al. 2011, chapter 6).

For the global RDA we report adjusted regression coefficients of multiple determination $\left(R_{\text {adj }}^{2}\right)$. In addition to the global RDA, we calculated variance partitioning (Borcard et al. 1992) to estimate the variance uniquely explained by the environment (EIS) or by space (S|E). Variance decomposition was performed if either the spatial or the environmental model were significant (Borcard et al. 2011). In these partial RDA models, covariables were included only if significant in either the spatial or the environmental model.

In summary, we determined for each genetic matrix (neutral microsatellites and SNPs, COI mtDNA, general stress loci, loci linked with fish predation, loci linked with land use and loci linked with parasite infection) the percentage of variance explained by: (i) the a priori selected set of environmental variables, (ii) the spatial matrix, and (iii) the conditional variance: the variance remaining after taking covariation into account (SIE and E|S). The entire set of analyses was performed for the whole set of 19 ponds, and separately for the coast $(n=9)$ and the inland populations $(n=10)$. For the neutral and the general stress-linked markers, the a priori selected set of environmental variables consisted of categorical fish density data, sample loadings for PCA1 and PCA2 of the parasite community and sample loadings for PCA1 and PCA2 of the land-use data. All RDA analyses were performed in R (v2.15.1; Oksanen 2011), using the rda and varpart functions of the vegan package (Oksanen 2011). In addition, the forward.sel function of the packfor package was used (Dray et al. 2006). MEM spatial eigenfunctions were computed using the create.MEM.model() function (Legendre et al. 2010) in the PCNM package (Legendre 2009).

\section{Results}

\section{Ancient (postglacial) demographic history of European Daphnia magna}

The maximum likelihood and the majority rule consensus tree based on Bayesian inference gave identical topologies and comparable statistical support for the identified clades with mtDNA haplotypes (Fig. S1, Supporting information). In general, the phylogenetic clades were statistically well supported and the European clade of D. magna was monophyletic, showing that all the haplotypes identified in our populations cluster in the same phylogenetic clade. The separation between the European and the non-European clades of D. magna
(Japan and USA) was significantly supported. As all haplotypes identified in Europe have a monophyletic origin and radiate from a common ancestor producing a star-like phylogeny, we considered all haplotypes observed in the Belgian populations as independent for what regards recent demographic history.

The total number of distinct COI haplotypes in Europe was 42 (29 excluding Scandinavia and populations located south of the Mediterranean Sea, Table S4, Supporting information), with $\mathrm{H} 18$ being the most abundant one throughout the European distributional range of D. magna. The haplotype diversity and richness we observed across the European range of D. magna was intermediate between the values observed in obligate parthenogenetic and obligate sexual crustacean species (e.g. Munoz et al. 2008, 2010). The Bayesian skyline plot (Fig. S2, Supporting information) drawn in Beast suggested a small $N_{\mathrm{e}}$ at European scale maintained constant through time. The mismatch distribution of haplotypes showed a unimodal distribution at European scale and a significant deviation (SSD $P<0.01$ ) of the observed distribution of haplotypes from the expected distribution in presence of demographic equilibrium (Fig. S3, Supporting information).

\section{Recent demographic history of Belgian D. magna}

$m t D N A$. The number of observed haplotypes in Belgium was 9, of which five were new haplotypes not described previously (De Gelas \& De Meester 2005; Table S3, Supporting information). The Belgian populations were mostly dominated by one haplotype, which was also the most common in Europe, and on average we observed two haplotypes per population in Belgium. The coastal populations were more homogeneous than the inland populations, with the latter showing a higher average number of haplotypes per population (Fig. 1) However, values of theta $(\theta$, haplotype diversity) did not differ significantly ( $t$-test, $P=0.08$ ) between inland and coastal populations (Table S4, Supporting information). Global $F_{\mathrm{ST}}$ was 0.44 . Pairwise population comparisons based on $F_{\mathrm{ST}}$ were significantly different from zero in $52 \%$ of the comparisons (Table S5, Supporting information). The populations showing a nonsignificant genetic differentiation were mostly dominated by the same, most widespread haplotype (H18). No isolation by distance was identified (Mantel $r=0.04 ; P=0.79$ ). Likewise, no significant spatial structure was detected in the RDA analysis.

Neutral microsatellite and SNP markers. Both microsatellites and SNP loci were in $\mathrm{H}-\mathrm{W}$ equilibrium in the populations analysed. Gametic linkage disequilibrium among pairs of loci ranged between $0.2 \%$ and $57 \%$ for 
microsatellites and between $0 \%$ and $9 \%$ for SNP loci of the total number of loci. The percentage of pairwise loci in gametic linkage disequilibrium varied among populations, with the highest values $(>30 \%)$ observed in Dana, TER1, ZW3 and ZW4 and Dana, MO, OM2, OM3 and ZW4 for microsatellites and SNPs, respectively (for population codes see Table S1, Supporting information).

Average $F_{\mathrm{ST}}$ value over all neutral loci was 0.12 both for SNPs and microsatellites. $F_{\mathrm{ST}}$ values for both markers were significantly larger than zero for all pairwise comparisons except for a few comparisons for SNPs (Table S6, Supporting information). The nonspatial inference of population genetic structure conducted with BAPS (Corander \& Marttinen 2006; Corander et al. 2008) confirmed the existence of a strong and significant differentiation among the populations analysed and indicated no admixture among populations, even among geographically adjacent populations. The only exceptions to this pattern were populations that are interconnected on the landscape. The BAPS plot clearly identified 11 as the best partition for the 19 populations analysed (Fig. 1) for microsatellite loci. Populations that were grouped in the same genetic clusters and therefore showed higher genetic similarity were either interconnected (OM1-OM2-OM3) or had a small sample size $(N<20$, Table S1, Supporting information). The latter are more prone to a sampling bias. For all populations from which we analysed 20 or more individuals, the genetic groups correspond entirely to the discrete populations sampled except for the set of interconnected ponds (Fig. 1). For SNP loci, BAPS identified 9 as the best partition for the 19 populations analysed (Fig. 1). This probably reflects lower statistical power to discriminate among groups due to the small sample size of some populations $(N<20$, Table S1, Supporting information) (Frantz et al. 2012).

Rare alleles (frequency below 0.05) were present at all microsatellite loci except for two loci, with an average of 1.1 rare alleles per locus per population. The rare alleles were shared across populations regardless of their geographic proximity and location in the landscape. Private microsatellite alleles were present in all populations, except for OM1 (the small sample size of this population may have contributed to this result), and ranged between $0.3 \%$ and $2.6 \%$ of the total number of alleles (over all loci) in the populations analysed. All our populations exhibited a pattern of heterozygosity that deviated significantly from mutation-drift equilibrium expectations both for microsatellites and SNPs, showing a significant excess of expected heterozygosity under the IAM mutation model (Table S7, Supporting information). The estimated current effective population size per population based on microsatellite loci was small and in most cases below 100 (Table S8, Supporting informa- tion). The gametic linkage disequilibrium between pairs of loci (microsatellites and SNPs) provides supporting evidence for the low effective population sizes of these populations.

\section{Environmental association analysis}

As compared to the genome scan study of Orsini et al. (2012) which focused on environmental gradients exerting strong selection pressure (fish predation, land-use intensity and parasite infection), here, we investigate how a wide array of biotic and abiotic variables contributes to population genomic structure in natural landscapes where D. magna occurs. To interpret the complexity typical of natural landscapes, we study patterns of genetic variation at markers with different mutation rates and include both neutral and nonneutral markers. We show that a number of environmental variables, in addition to the strong gradients previously studied, concur to drive genomic structure of natural populations in our study system.

Partial Mantel test. Some environmental variables covaried in the set of populations analysed (indicated in bold in Table S9, Supporting information) after Bonferroni correction: land-use intensity covaried with water quality, represented by Chla and suspended matter; temperature covaried with the abundance of macrophytes, probably as result of reduced penetration of solar radiation in macrophyte-rich ponds; transparency covaried with the amount of suspended matter in the water column; and parasite abundances covaried with other environmental variables, such as fish predation, macrophyte occurrence, and temperature. Using a partial Mantel test that allowed to control for geographic distance, we identified environmental variables covarying with changes in neutral and adaptive genetic variation across the landscape. We correlated environmental variables with different types of neutral markers (microsatellites, SNPs, COI mtDNA haplotypes), with specific stress loci (fish predation, land-use intensity and parasite infection as identified in the previous genome scan, Orsini et al. 2012) and with general stress loci (loci underlying multiple stress responses, Orsini et al. 2012). The environmental gradients that were the focus of the genome scan analysis (fish predation, land-use intensity and infection by the endoparasite $P$. ramosa) only correlated with specific stress loci, with the exception of land-use intensity that correlated with genetic variation also at neutral and general stress loci (Table 2). Correlation between land-use intensity and neutral genetic variation was previously shown for microsatellite and SNP loci (Orsini et al. 2012). Here we confirm those past results and, in addition, show that land-use intensity 
10 L. ORSINI ET AL.

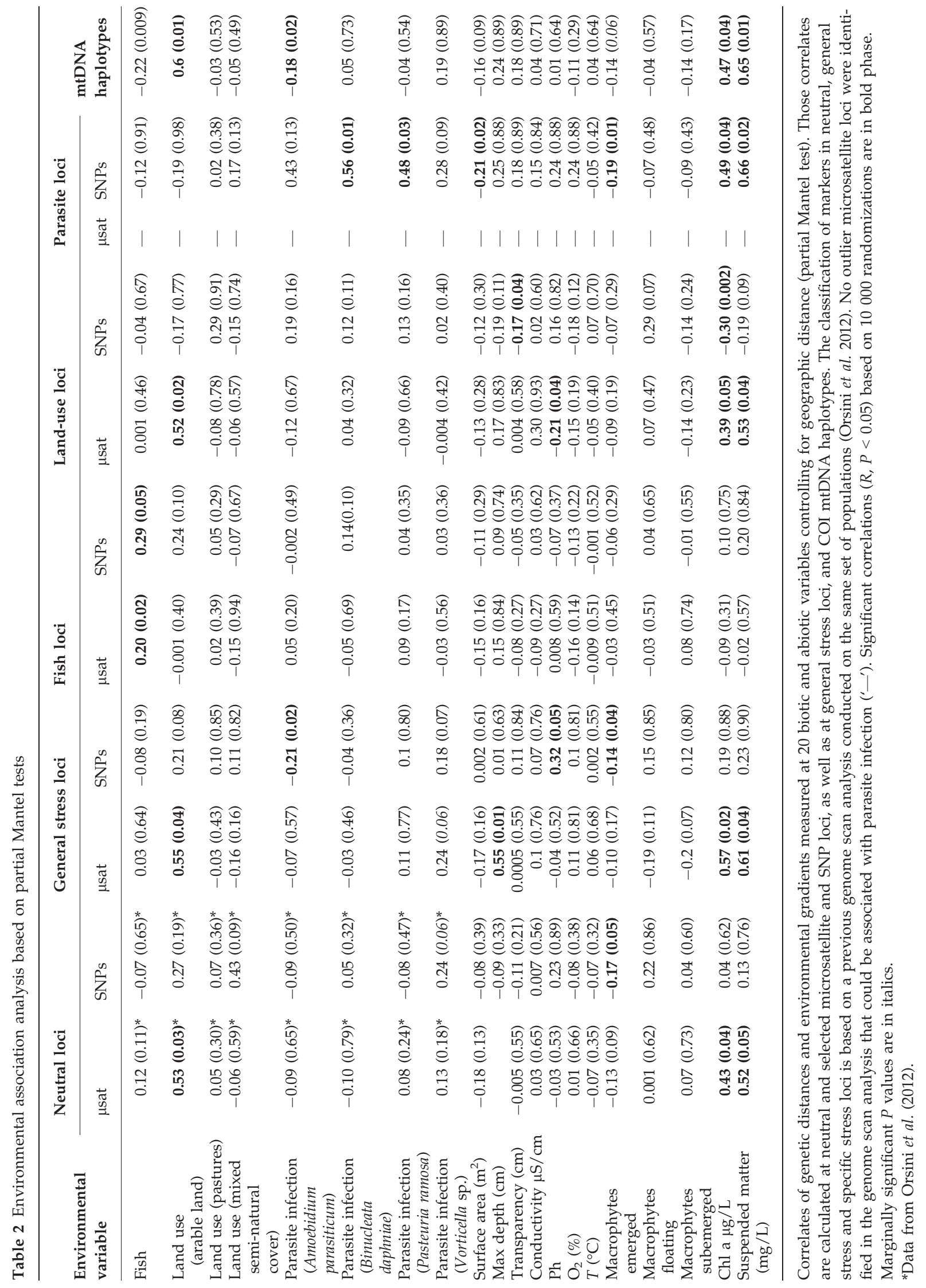


covaries with neutral variation at COI mtDNA haplotypes as well as with variation at microsatellite and SNP loci underlying general stress responses (Table 2). Environmental variables that significantly concur to drive genetic variation at loci linked with land-use intensity are Chla and suspended matter, as expected given the covariation of land-use intensity with the latter two environmental variables (Table S9, Supporting information). Macrophyte abundance and water quality contribute to shape genetic variation at loci associated with parasite infection (Table 2). General stress response loci are confirmed as such because they not only correlate with more than one strong environmental gradient (e.g. land-use intensity and fish predation), but also correlate with a number of additional environmental variables, including $\mathrm{pH}$ and presence of macrophytes (Table 2).

$R D A$. The RDA analysis adds yet another level of complexity to the analysis of environmental association performed with the partial Mantel test by allowing to identify the relative contribution of spatial and environmental signals to the population genomic structure of D. magna. The global RDA shows that a combination of space and environment drives genetic variation at neutral and general stress response microsatellite loci, as well as at SNP loci linked with fish predation (Table 3 and S10, Supporting information). The spatial component contributing to the genetic variation at neutral microsatellite markers is a combination of a large scale effect (dummy variable; coast versus inland) and local effect (MEM3-4), whereas the environmental variable that contributes to genetic variation is fish predation (Table S10, Supporting information). In addition to the spatial signal, land-use intensity and fish abundance drive genetic variation at microsatellite loci linked with general stress response, whereas genetic variation at general response SNP loci is explained by a combination of spatial signal and fish predation (Table S10, Supporting information). COI mtDNA variation is driven by a spatial component in combination with the presence of the parasite A. parasiticum (Table S10, Supporting information). The parasite $A$. parasiticum was also identified in the partial Mantel test to covary with genetic variation at mtDNA, indicating that the covariation with the parasite is not an artefact of the test performed and that this parasite may be spatially distributed in the landscape.

The global RDA analysis, conducted on the two clusters of populations separately, shows that the inland populations present similar patterns to the total set of populations for neutral and general stress response microsatellite loci. Here the spatial component is represented by MEM3-4, whereas the environmental component is a combination of fish predation (nearly significant) and land-use intensity (Table S10, Supporting information). The inland populations also show a significant environmental signal driving genetic variation at COI mtDNA (Table 3). A purely environmental signal drives genetic variation at microsatellite loci linked with two strong environmental gradients (fish predation and land use), as well as at COI mtDNA in the total set of populations analysed (Tables 3 and S10, Supporting information).

Following forward selection, a spatial signal remains significant only for neutral microsatellite and fish-linked SNP loci in the total set of populations, whereas a purely environmental signal remains significant for microsatellite and SNP loci linked with the respective strong environmental gradients: either fish predation or land-use intensity (Table 3). The RDA analysis identifies neither a spatial nor an environmental signal associated with the third strong environmental gradient studied in the previous genome scan and represented by parasite infection.

In general, a combination of environmental and spatial components explains a significant part of the genetic variation measured at microsatellite and SNP loci, although less well in the latter. Variation at COI mtDNA haplotypes is neither explained by an environmental nor by a spatial component, except for the covariation between mtDNA and the parasite A. parasiticum. In addition, coastal populations are not influenced by either spatial or environmental selection, one exception being land-use intensity, whereas the set of inland populations responds significantly to environmental selection (Table 3). In summary, except for the loci underlying specific stress response, whose genetic variation is explained by a purely environmental signal, genetic variation at other markers is driven by a combination of spatial and environmental components (Table 3), as the partial RDA tests show (Table 3). Partial RDA tests are either not significant or not applicable because of the double stop criterion adopted (Blanchet et al. 2008), with the exception of one test for neutral microsatellites (S|E). This suggests that spatial and environmental signals cannot be disentangled in most tests.

\section{Discussion}

Although much progress has been made in understanding causes and consequences of local and regional changes in genetic diversity of natural populations, it remains arduous to interpret patterns of genetic diversity and identify processes responsible for population genetic structure in the wild. Difficulties in interpreting patterns of genetic diversity in the wild derive from the 
12 L. ORSINI ET $A L$.

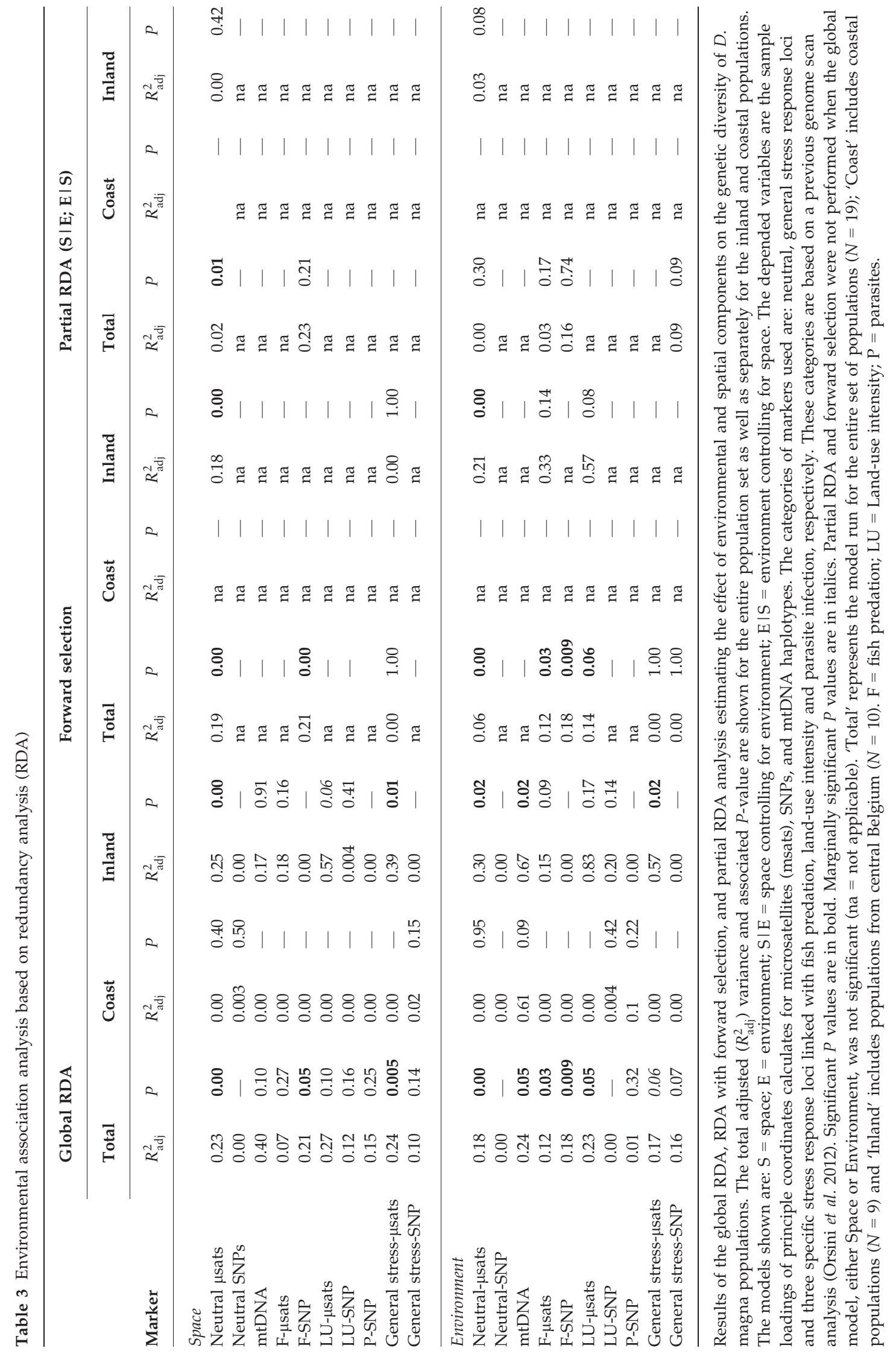


fact that similar patterns may be determined by different processes. For example processes such as adaptation, dispersal limitation and genetic drift, may result in similar patterns of genetic variation. Patterns of isolation by distance (IBD) have been often associated with a negative relationship between gene flow and geographic distance as a structuring force of population divergence (Hutchinson \& Templeton 1999). However, IBD may also be caused by spatial autocorrelation in environmental heterogeneity and associated selection (Sokal et al. 1989; Epperson 1990) or serial colonization (Sokal \& Oden 1978a,b; Sokal et al. 1989), thus complicating the identification of processes responsible for structuring population differentiation. In addition, processes driving population genetic structure in the wild do not act in isolation, but rather interact at the level of the landscape, complicating the identification of individual processes that determine patterns of population differentiation.

To identify the processes that drive population genomic structure in natural populations of D. magna, we studied patterns of population genetic structure at neutral marker and at markers linked with genes under selection, as identified in an earlier study on the same populations (Orsini et al. 2012). The analysis of patterns of genetic variation at neutral markers with different mutation rates allowed us to identify past as well recent demographic processes contributing to the present-day landscape genomic structure. The analysis of correlates between environmental variables and genetic variation at genome-wide level in D. magna identified changes in the environment that contribute to the genomic structure of D. magna in a regional landscape. In the following, we discuss these results.

\section{Patterns of neutral and adaptive genetic variation in the waterflea D. magna}

The combined analysis of markers with different mutation rates as well as the analysis of both neutral and adaptive variation, portrayed a complex combination of neutral and selective processes interacting to structure genomic variation of D. magna at the landscape level.

The ancient demographic history of the European D. magna as described by the phylogenetic inference of the COI mtDNA haplotypes indicates a monophyletic origin of the D. magna clade in central Europe. The starlike phylogeny of the central European clade suggests a unique founding event and parallel radiation of the different haplotypes. This is confirmed by the mismatch distribution analysis that presents a bell-shaped distribution of haplotypes at European scale reflecting the signature of past colonization events and absence of demographic equilibrium.
The analysis of recent demographic history, portrayed by the COI mtDNA haplotypes, SNP and microsatellite loci, indicates a strong and significant population divergence among all populations in our study system, including adjacent populations, suggestive of low levels of gene flow at the landscape level. The absence of a signal of isolation by distance at all markers indicates that genetic exchange among adjacent populations is not higher than among distant populations, and hence that gene flow is reduced among all populations. The inference of population genetic structure with microsatellite and SNP markers supports a strong population structure in our study system. These findings confirm past results in which strong genetic differentiation was observed also among adjacent populations (Vanoverbeke \& De Meester 1997; Haag et al. 2006). Yet, our levels of $F_{\mathrm{ST}}$ for diploid markers (both microsatellites and SNPs) are lower than the ones observed with allozymes in previous studies on the same species and involving some of the populations used here (Vanoverbeke et al. 2007). The levels of local and regional diversity we observe at microsatellite loci are, however, in the range reported for D. magna in Southern/Central Europe in a study analysing individuals hatched from resting eggs or sampled immediately after hatching (Walser \& Haag 2012). Our study, together with the one of Walser \& Haag (2012), is the first to apply genetics / genomics to Daphnia populations hatched from dormant eggs and therefore minimizes the confounding effects of clonal reproduction during the growing season. Most existing population genetic studies on Daphnia performed their analysis on active populations in which clonal reproduction predominates. During the growing season, clonal diversity can be very low within populations and this tends to increase among-population divergence (Vanoverbeke \& De Meester 2010). The use of resting egg banks may explain the lower levels of $F_{\mathrm{ST}}$ observed here.

The effective population size in the studied populations was maintained small after colonization. This assertion is supported by the analysis of historical effective population size (skyline plot) and the recent estimation of effective population size inferred with fast evolving markers (microsatellites). Together with the presence of strong gametic linkage disequilibrium between a number of pairwise neutral loci, this result indicates that the effective population size is maintained small through time. The persistent low effective population size associated with a relatively high $F_{\mathrm{ST}}$ among populations (Hartl \& Clark 2007) points towards low levels of gene flow at landscape level. High levels of $F_{\mathrm{ST}}$ could be explained by low effective population size associated with low levels of gene flow, which increases population differentiation. However, our populations 
exhibit low effective population size and we measure relatively high values of population differentiation. We therefore conclude that levels of gene flow must be low at landscape level.

In summary, the results for the neutral variation at landscape level indicate moderate (microsatellites and SNPs) to high (mtDNA) genetic differentiation among populations also at small spatial scale, low effective population size maintained through time, and low levels of gene flow at landscape level. Previous results (Orsini et al. 2012) and the analysis of adaptive variation in this study indicate that environmental selection plays a major role in driving genetic variation at candidate genome regions and to some extend affects neutral variation. We discuss in the following the role played by the environment in our study system.

\section{The role of the environment in the landscape genomic structure of D. magna}

Understanding how the genome variation in natural populations may be driven by environmental changes is a challenging task. Since the establishment of the field of landscape genetics (Manel et al. 2003), an explicit spatial component has been increasingly applied in the analysis of neutral population genetic structure (Orsini et al. 2008; Munshi-South 2012). However, most population genetic studies do not pay much attention to environmental gradients in the landscape, and rarely do they consider more than one gradient. In addition, only recently have the environmental correlates with adaptive genetic variation been measured in natural populations (Manel et al. 2010a,b; Orsini et al. 2012). Studies that analyse in parallel neutral and adaptive genetic variation in a landscape context and/or in relation to environmental gradients are generally limited to a few model taxa (e.g. Stranger et al. 2011). This is because such an integrated approach requires a large set of genomic tools as well as a deep knowledge of the landscape where the populations/species occur. Here, we analyse both neutral and adaptive genetic variation (variation at loci hitchhiking with genes under selection) and correlate such variation with landscape and environmental variables in an attempt to identify processes of adaptation in complex natural landscapes. It is to be expected that with the higher accessibility of genomic tools to nonmodel taxa this integrated approach will become increasingly applied in nonmodel taxa.

A previous genome scan study (Orsini et al. 2012) allowed us to identify genome regions and putative candidate loci linked with three strong ecological gradients known to exert selection pressure on Daphnia populations (fish predation, land-use intensity and parasite infection). Natural landscapes, however, are a complex mosaic of biotic and abiotic variables that contribute to drive population genomic structure. Building in complexity on our previous genome scan, here we investigate how the genome structure changes along environmental gradients as measured in a set of 20 abiotic and biotic variables. The environmental association analysis involving the 20 variables provides us with two important observations. First, at a first glance it seems that a combination of spatial and environmental signals drives population genomic structure in our study system both at neutral and adaptive genetic variation. However, when a forward selection is applied using as explanatory variables the strong ecological gradients studied in the previous genome scan, it appears that the environmental signal becomes more important than the spatial signal, at least for loci linked with genes under selection. A combination of a spatial and environmental signal remains significant in driving genetic variation in the rest of the genome. This result underlies the key role played by environmental selection in our study system. It also indicates an important methodological limitation: in natural systems for which knowledge of the ecological background is scarce and the major environmental gradients have not been identified, one may underestimate the effect of the environment in driving population genomics structure. The second important observation comes from the analysis of the inland and coastal populations as separate clusters. By analysing these populations as two separate sets, we observe that the environment, sometimes combined with a spatial signal, exerts a significant selective pressure on the inland more than on the coastal populations. This difference probably reflects key differences in ecological characteristics of these two population sets. This asserts the importance of analysing patterns of genetic variation at different spatial scales, as suggested (Manel et al. 2010b). Here, we reinforce that not only the spatial scale but the unique ecological and environmental characteristics of natural systems of the same specie at different spatial scales and in different regions may play a strong role in determining the adaptive responses of natural systems to environmental changes.

\section{The role of selection in the population genomic} structure of Daphnia magna

The parallel study of demographic and adaptive patterns in a landscape genomics context allowed us to identify some of the processes likely responsible for the present-day population genomic structure of D. magna. Our results suggest two alternative scenarios that lead to the present-day population genetic structure: 
environmental selection combined either with persistent founder effect (Boileau et al. 1992) or genetic drift (De Meester et al. 2002).

Multiple evidence suggests that a stochastic process such as genetic drift is not likely to be solely responsible for population genetic structure in our system. The persistent small effective population size maintained through several generations associated with low levels of gene flow, together with the presence of gametic linkage disequilibrium, and the ubiquity of rare and private alleles suggest that other processes (e.g. selection) are at work in our system.

The theory for persistent founder effect formalized by Boileau et al. (1992) states that in organisms like zooplankton, which combine the potential to colonize whole habitats from a few propagules with the capacity for rapid population growth, allele frequencies established during initial founder events are resistant to decay for several thousands of generations, even with substantial rates of gene flow. As the initial colonizers have the capacity to increase rapidly to very high numbers, saturating the habitat in a few generations, the impact of subsequent migrants is strongly diluted. Such persistent founder effects effectively reflect priority effects arising from the gross disparity in the numbers of residents and immigrants, resulting in a low probability of random increase in migrant genotypes due to drift alone. The theory of Boileau et al. (1992) assumes that gene flow will eventually dilute the founder effect and bring the system to equilibrium. Depending on the effective size of the population, founder effects will decay at different speed. With moderate to high levels of gene flow, founder events will persist for many generation only if the effective population size is large (Crow \& Aoki 1984; Boileau et al. 1992).

De Meester et al. (2002) extended the theory of Boileau et al. (1992) suggesting that local genetic adaptation plays a strong role and can enhance founder effects making them more resistant to decay. According to De Meester et al. (2002), rapid growth rate of zooplankton populations results in the monopolization of resources by descendants of the first immigrant (similar to Boileau et al. 1992), enhanced further by the fact that the resident population genetically adapts to the local environment. This scenario is called 'monopolization' to refer to the fact that resident populations are better adapted to local environmental conditions than immigrants and tend to monopolize the local resources. Local genetic adaptation reduces the establishment success of immigrants, effectively reducing gene flow among populations. Indeed, past research has shown that after the colonization of pristine habitats, the establishment success of immigrants is low and that effective gene flow is probably much lower than the dispersal rate (Louette et al. 2007).

The predictions of the monopolization hypothesis have not been, so far, translated in population genetic terms. Here we set out to interpret the genetic patterns observed in D. magna in terms of the monopolization hypothesis. One of the most important assumptions of the monopolization hypothesis is that once the local genetic pool is established, local genetic adaption reduces gene flow among populations through a reduced establishment success of immigrants, maintaining this situation for much longer than expected under neutrality. As the monopolization of resources happens independently in the different habitats in the landscape, gene flow is reduced among all habitat patches, including the adjacent ones. Reduced gene flow among populations in the landscape regardless of their geographic proximity results in strongly structured populations and the absence of a signal of isolation by distance. Indeed, a moderate to high level of gene flow will invariably lead to patterns of IBD at regional scale. Our analyses clearly indicate strong landscape genetic structure (pairwise $F_{\mathrm{ST}}$ and BAPS plots) and the absence of a signal of isolation by distance. This does not exclude that a local or regional spatial signal other than a linear correlation between genetic and geographic distance still influences population genetic structure, as shown by the RDA analysis. We also observe small (in the order of 100) and constant $N_{\mathrm{e}}$ through time associated with relatively high $F_{\mathrm{ST}}$. Thus, multiple evidence supports the hypothesis that monopolization may play a key role in shaping population genetic structure in our study system.

Given the small $N_{\mathrm{e}}$ observed in our populations one may expect inbreeding, resulting in a high number of homozygous genotypes and a low local genetic diversity. Instead, we observe in our populations higher genetic diversity than expected (excess in expected heterozygosity, Table S7, Supporting information). We see two alternative hypotheses that can explain the patterns of heterozygosity we observe: a recent bottleneck or overdominance. Given that some of our ponds are man-made and we can place their establishment at 100 years ago or more, the fact that we can detect signals of a recent bottleneck suggests that our populations may go through extinction-colonization events cyclically. Extinction-colonization dynamics are expected with the type of landscape we analyse here. The study populations mostly inhabit small, sometimes artificial, farmland ponds. Because of their size, these ponds may be relatively short-lived (a few decades to a few hundred years) as they may evolve into marshland or may be filled because of a change in land use. Further studies are needed to confirm the hypothesis of extinction-colonization 
dynamics. The hypothesis of overdominance is in apparent disagreement with past studies. Inbreeding has been observed in cyclical parthenogens (Deng \& Lynch 1997), including D. magna populations (Haag et al. 2002). However, those populations were from ephemeral habitats (rockpools) in which resting egg banks are absent or poorly developed. With evidence supporting low immigration success in our study system, the patterns we observe can be explained by the buffering effect of the dormant egg banks that enriches the local genetic pool re-introducing alleles from past growing seasons.

We conclude that selection plays a key role in driving landscape genomic structure in the cyclical parthenogen D. magna. Importantly, selection directly impacts adaptive variation in the studied landscape, reflected by significant patterns of isolation by environment for markers linked with loci under selection. In addition, both the low levels of gene flow and low effective population sizes are mediated by selection acting as a strong filter on clonal diversity within populations during the growing season. The overall pattern we observe is one of the environmental selections probably combined with monopolization, where a restricted number of effective migrants have a large impact on the landscape genetic structure.

\section{Acknowledgements}

LO and JVO are postdoctoral research fellows with the Fund for Scientific Research, Flanders (FWO). This research was financially supported by KU Leuven Research Fund grants GOA/2008/6, PF/2010/7 and FWO project G.0468.10. This work is part of the STRESSFLEA project of the European Science Foundation EUROCORES Programme EuroEEFG. The funders had no role in study design, data collection and analysis, decision to publish or preparation of the manuscript.

\section{References}

Adamowicz SJ, Petrusek A, Colbourne JK, Hebert PD, Witt JD (2009) The scale of divergence: a phylogenetic appraisal of intercontinental allopatric speciation in a passively dispersed freshwater zooplankton genus. Molecular Phylogenetics and Evolution, 50, 423-436.

Balanya J, Oller JM, Huey RB, Gilchrist GW, Serra L (2006) Global genetic change tracks global climate warming in Drosophila subobscura. Science, 313, 1773-1775.

Belkhir K, Borsa P, Goudet J, Bonhomme F (2004) GENETIX 4.05, logiciel sous WindowsTM pour la génétique des populations. Laboratoire Génome, Populations. Interactions CNRS UMR 5000, Université de Montpellier II, Montpellier, France.

Blanchet FG, Legendre P, Borcard D (2008) Forward selection of explanatory variables. Ecology, 89, 2623-2632.

Bohonak AJ (2002) IBD (Isolation By Distance): a program for analyses of isolation by distance. Journal of Heredity, 93, 153-154.

Boileau MG, Hebert PDN, Schwartz SS (1992) Non-equilibrium gene frequency divergence: persistent founder effects in natural populations. Journal of Evolutionary Biology, 5, 25-39.

Bonin A, Taberlet P, Miaud C, Pompanon F (2006) Explorative genome scan to detect candidate loci for adaptation along a gradient of altitude in the common frog (Rana temporaria). Molecular Biology and Evolution, 23, 773-783.

Borcard D, Legendre P, Drapeau P (1992) Partialling out the spatial component of ecological variation. Ecology, 73, 1045-1055.

Borcard D, Gillet F, Legendre P (2011) Numerical Ecology with $R$. Springer, New York, Dordrecht, London, Heidelberg.

Colbourne JK, Pfrender ME, Gilbert D et al. (2011) The ecoresponsive genome of Daphnia pulex. Science, 331, 555-561.

Coors A, Decaestecker E, Jansen M, De Meester L (2008) Pesticide exposure strongly enhances parasite virulence in an invertebrate host model. Oikos, 117, 1840-1846.

Coors A, Vanoverbeke J, De Bie T, De Meester L (2009) Land use, genetic diversity and toxicant tolerance in natural populations of Daphnia magna. Aquatic Toxicology, 95, 71-79.

Corander J, Marttinen P (2006) Bayesian identification of admixture events using multi-locus molecular markers. Molecular Ecology, 15, 2833-2843.

Corander J, Waldmann P, Marttinen P, Sillanpaa MJ (2004) BAPS 2: enhanced possibilities for the analysis of genetic population structure. Bioinformatics, 20, 2363-2369.

Corander J, Sirén J, Arjas E (2008) Bayesian spatial modeling of genetic population structure. Computational Statistics, 23, 111-129.

Cornuet JM, Luikart G (1997) Description and power analysis of two tests for detecting recent population bottlenecks from allele frequency data. Genetics, 144, 2001-2014.

Cousyn C, De Meester L, Colbourne JK, Brendonck L, Verchuren D, Volckaert F (2001) Rapid, local adaptation of zooplankton behavior to changes in predation pressure in the absence of neutral genetic changes. PNAS, 98, 6256-6260.

Crow JF, Aoki K (1984) Group selection for a polygenic behavioral trait: estimating the degree of population subdivision. Proceedings of the National Academy of Sciences of the United States of America, 81, 6073-6077.

Davey JW, Hohenlohe PA, Etter PD, Boone JQ, Catchen JM, Blaxter ML (2011) Genome-wide genetic marker discovery and genotyping using next-generation sequencing. Nature Review Genetics, 12, 499-510.

De Bie T, De Meester L, Brendonck L et al. (2012) Body size and dispersal mode as key traits determining metacommunity structure of aquatic organisms. Ecology Letters, 15, 740-747.

De Gelas K, De Meester L (2005) Phylogeography of Daphnia magna in Europe. Molecular Ecology, 14, 753-764.

De Meester L, Gomez A, Okamura B, Schwenk K (2002) The Monopolization Hypothesis and the dispersal-gene flow paradox in aquatic organisms. Acta Oecologica, 23, 121-135.

Decaestecker E, Gaba S, Raeymaekers J et al. (2007) Host-parasite Red Queen dynamics archived in pond sediment. Nature, 450, 870-874.

Declerck SAJ, Coronel JS, Legendre P, Brendonck L (2011) Scale dependency of processes structuring metacommunities of cladocerans in temporary pools of High-Andes wetlands. Ecography, 34, 296-305. 
Deng HW, Lynch M (1997) Inbreeding depression and inferred deleterious-mutation parameters in Daphnia. Genetics, 147, 147-155.

Dieringer D, Schlötterer C (2003) Microsatellite analyser (MSA): a platform independent analysis tool for large microsatellite data sets. Molecular Ecology Notes, 3, 167-169.

Dray S, Legendre P, Blanchet G (2006) Compiled package containing the functions "forward.sel" and "forward.sel.par"packfor", repos="http:/ /R-Forge.R-project.org.

Drummond A, Rambaut A (2002-2006) BEAST v1.4 Bayesian Evolutionary Analysis Sampling Trees. University of Oxford, Oxford.

Drummond AJ, Rambaut A, Shapiro B, Pybus OG (2005) Bayesian coalescent inference of past population dynamics from molecular sequences. Molecular Biology and Evolution, 22, 1185-1192.

Duvernell DD, Lindmeier JB, Faust KE, Whitehead A (2008) Relative influences of historical and contemporary forces shaping the distribution of genetic variation in the Atlantic killifish, Fundulus heteroclitus. Molecular Ecology, 17, 1344-1360.

Ebert D (2005) Ecology, Epidemiology, and Evolution of Parasitism in Daphnia. National Library of Medicine (US), National Center for Biotechnology, Bethesda, MD.

Eckert AJ, Bower AD, Gonzalez-Martinez SC, Wegrzyn JL, Coop G, Neale DB (2010) Back to nature: ecological genomics of loblolly pine (Pinus taeda, Pinaceae). Molecular Ecology, 19, 3789-3805.

Epperson BK (1990) Spatial Autocorrelation of Genotypes Under Directional Selection. Genetics, 124, 757-771.

Excoffier L, Laval G, Schneider S (2005) Arlequin ver. 3.0: an integrated software package for population genetics data analysis. Evolutionary Bioinformatics Online, 1, 47-50.

Frantz AC, Bertouille S, Eloy MC, Licoppe A, Chaumont F, Flamand MC (2012) Comparative landscape genetic analyses show a Belgian motorway to be a gene flow barrier for red deer (Cervus elaphus), but not wild boars (Sus scrofa). Molecular Ecology, 21, 3445-3457.

Guindon S, Dufayard JF, Lefort V, Anisimova M, Hordijk W, Gascuel O (2010) New algorithms and methods to estimate maximum-likelihood phylogenies: assessing the performance of PhyML 3.0. Systematic Biology, 59, 307-321.

Haag CR, Hottinger JW, Riek M, Ebert D (2002) Strong inbreeding depression in a Daphnia metapopulation. Evolution, 56, 518-526.

Haag CR, Riek M, Hottinger JW, Pajunen VI, Ebert D (2006) Founder events as determinants of within-island and amongisland genetic structure of Daphnia metapopulations. Heredity, 96, 150-158.

Hairston JNG, Lampert W, Caceres CE et al. (1999) Rapid evolution revealed by dormant eggs. Nature, 401, 446.

Hall TA (1999) BioEdit: a user-friendly biological sequence alignment editor and analysis program for Windows 95/98/ NT. Nucleic Acids Symposium Series, 41, 95-98.

Hartl DL, Clark AG (2007) Principles of Population Genetics, 4th edn. Sinauer and Associates, Sunderland, MA.

Hutchinson WF, Templeton AR (1999) Correlation of pairwise genetic and geographic distance measures: inferring the relative influences of gene flow and drift on the distribution of genetic variability. Evolution, 53, 1898-1914.

Jansen B, Geldof S, De Meester L, Orsini L (2011) Isolation and characterization of microsatellite markers in the waterflea Daphnia magna. Molecular Ecology Resources, 11, 418-421.
Jones FC, Grabherr MG, Chan YF et al. (2012) The genomic basis of adaptive evolution in threespine sticklebacks. Nature, 484, 55-61.

Lampert W (2011) Daphnia: Development of a Model Organism in Ecology and Evolution. International Ecology Institute, Oldendorf.

Legendre P (2009) PCNM: PCNM spatial eigenfunction and principal coordinate analyses. $\mathrm{R}$ Forge. $\mathrm{R}$ package version 1.9. https://r-forge.r-project.org/.

Legendre P, Fortin MJ (2010) Comparison of the Mantel test and alternative approaches for detecting complex multivariate relationships in the spatial analysis of genetic data. Molecular Ecology Resources, 10, 831-844.

Legendre P, Gallagher ED (2001) Ecologically meaningful transformations for ordination of species data. Oecologia, 129, 271-280.

Legendre P, Legendre L (2012) Numerical Ecology, 3rd edn. Elsevier, Quebec, Canada.

Legendre P, De Caceres M, Borcard D (2010) Community surveys through space and time: testing the space-time interaction in the absence of replication. Ecology, 91, 262-272.

Librado P, Rozas J (2009) DnaSP v5: a software for comprehensive analysis of DNA polymorphism data. Bioinformatics, 25, 1451-1452.

Louette G, Vanoverbeke J, Ortells R, De Meester L (2007) The founding mothers: the genetic structure of newly established Daphnia populations. Oikos, 116, 728-741.

Luikart G, Cornuet JM (1998) Empirical evaluation of a test for identifying recently bottlenecked populations from allele frequency data. Conservation Biology, 12, 228-237.

Luikart G, Phillip R, Tallmon D, Jordan S, Taberlet P (2003) The power and promise of population genomics: from genotyping to genome typing. Nature Reviews Genetics, 4, 981-994

Manel S, Schwartz MK, Luikart G, Taberlet P (2003) Landscape genetics: combining landscape ecology and population genetics. Trends in Ecology and Evolution, 18, 189-197.

Manel S, Joost S, Epperson BK et al. (2010a) Perspectives on the use of landscape genetics to detect genetic adaptive variation in the field. Molecular Ecology, 19, 3760-3772.

Manel S, Poncet BN, Legendre P, Gugerli F, Holderegger R (2010b) Common factors drive adaptive genetic variation at different spatial scales in Arabis alpina. Molecular Ecology, 19, 3824-3835.

Marcus NH (1990) Calanoid copepod, cladoceran, and rotifereggs in sea-bottom sediments of northern Californian coastal waters: identification, occurrence and hatching. Marine Biology, 105, 413-418.

Metzker ML (2010) Sequencing technologies - the next generation. Nature Reviews Genetics, 11, 31-46.

Michels H (2008) Micro-Evolutionary Response of Daphnia magna to Changes in Biotic Stress Associated with Habitat Degradation and Restoration of a Shallow Lake. Doctoral thesis in Biology, Laboratory of Aquatic Ecology and Evolutionary Biology, University of Leuven.

Miner BE, De Meester L, Pfrender ME, Lampert W, Hairston Jr NG (2012) Linking genes to communities and ecosystems: Daphnia as an ecogenomic model. Proceedings. Biological Sciences, 279, 1873-1882.

Morin PA, Luikart G, Wayne RK, group Sw (2004) SNPs in ecology, evolution and conservation. Trends in Ecology and Evolution, 19, 208-216. 
Munoz J, Gomez A, Green AJ, Figuerola J, Amat F, Rico C (2008) Phylogeography and local endemism of the native Mediterranean brine shrimp Artemia salina (Branchiopoda: Anostraca). Molecular Ecology, 17, 3160-3177.

Munoz J, Gomez A, Green AJ, Figuerola J, Amat F, Rico C (2010) Evolutionary origin and phylogeography of the diploid obligate parthenogen Artemia parthenogenetica (Branchiopoda: Anostraca). PLoS One, 5, e11932.

Munshi-South J (2012) Urban landscape genetics: canopy cover predicts gene flow between white-footed mouse (Peromyscus leucopus) populations in New York City. Molecular Ecology, 21, 1360-1378.

Namroud MC, Beaulieu J, Juge N, Laroche J, Bousquet J (2008) Scanning the genome for gene single nucleotide polymorphisms involved in adaptive population differentiation in white spruce. Molecular Ecology, 17, 3599-3613.

Nei M (1972) Genetic distance between populations. American Naturalist, 106, 283-292.

Nosil P, Funk DJ, Ortiz-Barrientos D (2009) Divergent selection and heterogeneous genomic divergence. Molecular Ecology, $18,375-402$.

Oksanen J (2011) Multivariate Analysis of Ecological Communities in $\mathrm{R}$ : vegan tutorial. http://cc.oulu.fi/ jarioksa/opetus/metodi/vegantutor.pdf.

Onbe T (1978) Sugar flotation method for sorting the resting eggs of marine cladocerans and copepods from sea-bottom sediment. Bulletin Japanese Society Scientific Fisheries, 44, 1411.

Orsini L, Corander J, Alasentie A, Hanski I (2008) Genetic spatial structure in a butterfly metapopulation correlates better with past than present demographic structure. Molecular Ecology, 17, 2629-2642.

Orsini L, Jansen M, Souche EL, Geldof S, De Meester L (2011) Single nucleotide polymorphism discovery from expressed sequence tags in the waterflea Daphnia magna. BMC Genomics, 12, 309.

Orsini L, Spanier KI, De Meester L (2012) Genomic signature of natural and anthropogenic stress in wild populations of the waterflea Daphnia magna: validation in space, time and experimental evolution. Molecular Ecology, 21, 2160-2175.

Ray N, Currat M, Excoffier L (2003) Intra-Deme Molecular Diversity in Spatially Expanding Populations. Molecular Biology and Evolution, 20, 76-86.

Rogers AR, Harpending H (1992) Population growth makes waves in the distribution of pairwise genetic differences. Molecular Biology and Evolution, 9, 552-569.

Ronquist F, Huelsenbeck JP (2003) MrBayes 3: Bayesian phylogenetic inference under mixed models. Bioinformatics, 19, 1572-1574.

Rousset F (2008) Genepop'007: a complete reimplementation of the Genepop software for Windows and Linux. Molecular Ecology Resources, 8, 103-106.

Routtu J, Jansen B, Colson I, De Meester L, Ebert D (2010) The firstgeneration Daphnia magna linkage map. BMC Genomics, 11, 508.

Schneider S, Excoffier L (1999) Estimation of demographic parameters from the distribution of pairwise differences when the mutation rates vary among sites: application to human mitochondrial DNA. Genetics, 152, 1079-1089.

Slatkin M, Hudson RR (1991) Pairwise comparisons of mitochondrial DNA sequences in stable and exponentially growing populations. Genetics, 129, 555-562.
Sokal RR, Oden NL (1978a) Spatial autocorrelation in biology. 1. Methodology. Biological Journal Linnean Society, 10, 199-228.

Sokal RR, Oden NL (1978b) Spatial autocorrelation in biology. 2. Some biological implications and four applications of evolutionary and ecological interest. Biological Journal Linnean Society, 10, 229-249.

Sokal RR, Jacquez GM, Wooten MC (1989) Spatial Autocorrelation Analysis of Migration and Selection. Genetics, 121, 845-855.

Storz JF (2005) Using genome scans of DNA polymorphism to infer adaptive population divergence. Molecular Ecology, 14, 671-688.

Stranger BE, Stahl EA, Raj T (2011) Progress and promise of enome-wide association studies for human complex trai genetics. Genetics, 187, 367-383.

Thompson JD, Higgins DG, Gibson TJ (1994) CLUSTAL W: improving the sensitivity of progressive multiple sequence alignment through sequence weighting, position-specific gap penalties and weight matrix choice. Nucleic Acids Research, 22, 4673-4680.

Turner TL, Bourne EC, Von Wettberg EJ, Hu TT, Nuzhdin SV (2010) Population resequencing reveals local adaptation of Arabidopsis lyrata to serpentine soils. Nature Genetics, 42, 260-263.

Van Doorsaler W, Vanoverbeke J, Duvivier C et al. (2009) Local adaptation to higher temperatures reduces immigration success of genotypes from a warmer region in the water flea Daphnia. Global Change Biology, 15, 3046-3055.

Vangestel C, Mergeay J, Dawson DA, Callens T, Vandomme V, Lens L (2012) Genetic diversity and population structure in contemporary house sparrow populations along an urbanization gradient. Heredity, 109, 163-172.

Vanoverbeke J, De Meester L (1997) Among-population genetic differentiation in the cyclical parthenogen Daphnia magna (Crustacea, Anomopoda) and its relation to geographic distance and conal diversity. Hydrobiologia, 360, 135-142.

Vanoverbeke J, De Meester L (2010) Clonal erosion and genetic drift in cyclical parthenogens - the interplay between neutral and selective processes. Journal of Evolutionary Biology, 23, 997-1012.

Vanoverbeke J, De Gelas K, De Meester L (2007) Habitat size and the genetic structure of a cyclical parthenogen, Daphnia magna. Heredity, 98, 419-426.

Walser B, Haag CR (2012) Strong intraspecific variation in genetic diversity and genetic differentiation in Daphnia magna: the effects of population turnover and population size. Molecular Ecology, 21, 851-861.

Wang JI (2009) A new method for estimating effective population sizes from a single sample of multilocus genotypes. Molecular Ecology, 18, 2148-2164.

Wang Z, Gerstein M, Snyder M (2009) RNA-Seq: a revolutionary tool for transcriptomics. Nature Reviews Genetics, 10, 57-63.

L.O. conceived the study, performed the population genomic analyses and wrote the paper. J.M. performed the RDA analyses and contributed to the manuscript writing. J.V. and L.D.M. contributed to conceptual discussions and paper writing. 


\section{Data accessibility}

mtDNA COI sequencesare deposited in GenBank, AN: JQ279821-JQ280299.

Genotypes for microsatellite and SNP loci, as well as a fasta file containing the alignment of the COI mtDNA sequences are deposited in the DRYAD databank: doi:10.5061/dryad.5g4r0.

Samples location and complementary data analyses are uploaded as online supplemental material.

\section{Supporting information}

Additional supporting information may be found in the online version of this article.

Fig. S1 Phylogenetic tree of the mtDNA COI haplotypes.

Fig. S2 Skyline plot.

Fig. S3 Mismatch distribution of COI mtDNA haplotypes in Europe.
Table S1 Populations and environmental data.

Table S2 mtDNA COI amplification and sequencing primers.

Table S3 mtDNA haplotypes used in the phylogenetic analysis.

Table S4 Haplotype statistics for COI mtDNA.

Table S5 Pairwise $\mathrm{F}_{\mathrm{ST}}$ values in the set of populations analyzed based on COI mtDNA.

Table S6 Pairwise $F_{S T}$ values in the set of populations analyzed based on neutral microsatellite and SNP markers.

Table S7 Test of mutation-drift equilibrium for microsatellites and SNPs.

Table S8 $N_{e}$ per population.

Table S9 Partial Mantel test on environmental variables.

Table S10 Environmental association analysis based on RDA. 